\title{
LETTER
}

\section{Numerical analysis of fringe patterns recorded in holographic interferometry using high-order ambiguity function}

\author{
Sai Siva Gorthi and Pramod Rastogi* \\ Applied Computing and Mechanics Laboratory, Ecole Polytechnique Fédérale de \\ Lausanne, 1015 Lausanne, Switzerland.
}

(Feb 2009)

\begin{abstract}
This Letter introduces a new approach for the demodulation of fringe patterns recorded in holographic interferometry using high-order ambiguity function (HAF). The proposed approach is capable of retrieving the phase from a single fringe pattern. The main advantage of this approach is that it directly provides an estimation of the continuous phase distribution and thereby avoids the necessity of using cumbersome 2-D phase unwrapping procedure. This method first computes the discrete-time analytic signal of the recorded fringe pattern. Then, by modeling this analytic signal as a polynomial phase signal embedded in additive complex white Gaussian noise, a parametric estimation procedure based on HAF is employed to directly estimate the unwrapped phase distribution. Numerical simulations and experimental results demonstrate the potential of the proposed approach.
\end{abstract}

Keywords: Holographic interferometry, fringe analysis, phase measurement, parametric estimation, high-order ambiguity function

\section{Introduction}

In interferometric applications, accurate estimation of phase maps from the recorded interferograms plays a vital role in deciding the accuracy of measurements, such as displacements, shapes or surface deformations. Over the last three decades, several phase estimation methods (often referred to as fringe analysis techniques) have been developed. Broadly they can be categorized as spatial and temporal analysis techniques. Their efficient and successful application require the presence of a spatial carrier for spatial methods, and acquisition of a number of phase-shifted interferograms for temporal methods. Moreover, most of these methods, either temporal or spatial, generate wrapped phase maps, which need to be further unwrapped in order to obtain an estimation of the true phase map.

The most simple and widely used technique for the spatial analysis of a carrier fringe pattern is the Fourier Transform Method, proposed by Takeda et al. (1). Fourier Transform is well suited for the analysis of stationary signals. In practice, the parameters of many of the interferometric fringe patterns are space-varying. These patterns represent non-stationary signals and analysis of such signals by simple Fourier transform provides results which are prone to important errors. During recent years, this realization has motivated researchers to propose the use of advanced spatial analysis methods such as those based on Windowed Fourier

\footnotetext{
*Corresponding author. Email: pramod.rastogi@epfl.ch
} 
transform (WFT) or Gabor transform (2, 3), Dilating Gabor transform (4), 1-D and 2-D Wavelet transforms $(5-9)$, Wigner-Ville distribution $(10,11)$ and regularized phase tracking (RPT) (12).

This Letter introduces a new approach for phase retrieval in holographic interferometry that directly provides unwrapped phase distribution from a single fringe pattern. The proposed approach relies on a signal processing technique, high-order ambiguity function (HAF), also referred to as polynomial phase transform (PPT) (13), developed for the parametric analysis of a particular class of 1-D time signals. Though it is applied in pulse compression radar systems and other fields, to the best of our knowledge this is the first time that it is proposed for fringe pattern analysis in interferometry.

\section{HAF-based fringe analysis in holographic interferometry}

A simple mathematical representation of the recorded holographic interference fringe pattern is given by

$$
I(x, y)=I_{0}\{1+\gamma(x, y) \cos [\phi(x, y)]\}
$$

where $I, I_{0}, \gamma$ and $\phi$ represent the recorded intensity, background intensity, fringe visibility and the phase term respectively, at the pixel $(x, y)$. While employing spatial analysis techniques, the phase in the argument of the cosine function is commonly expressed as $\phi(x, y)=\phi_{c}(x, y)+\phi_{d}(x, y)$ where $\phi_{c}(x, y)$ corresponds to the phase of the carrier added and $\phi_{d}(x, y)$ represents the deformation phase or the interference phase to be estimated. Even when the spatial carrier is added, it is not uncommon to be confronted with the fringe patterns similar to the one shown in Fig. 1a. This kind of a pattern represents a non-stationary signal in which the parameters associated with it, especially the frequency, vary considerably from one location to the other. As mentioned in introduction, conventional analysis techniques are not best suited to be applied for accurate phase estimation of these patterns. The analytic version of this kind of signals is referred to as polynomial phase signals, and parametric method based on HAF can provide accurate phase estimation. Eliminating the d.c. term from Eq.(1) using high-pass filtering results in:

$$
I^{\prime}(x, y)=a(x, y) \cos [\phi(x, y)]
$$

Note that with the help of any of the fringe normalization techniques (14-16), from Eq.(1) we can even get directly:

$$
I^{\prime}(x, y)=\cos [\phi(x, y)]
$$

First, we apply real to analytic signal conversion (17) on the Eq.(2) to obtain the analytic signal of the form:

$$
z(x, y)=a(x, y) \exp [j \phi(x, y)]
$$

Now one option could be to calculate phase from $z(x, y)$ using arctan function. Phase calculated in this manner is often noisy and on top of it, it is bound to be wrapped (phase values limited in the range of $-\pi$ to $+\pi$ ). The reason for phase map calculated directly from $z(x, y)$ to be noisy is that the latter contains noise 
inherited from the fringe pattern. Thus it is appropriate to represent the analytic signal of the fringe pattern as:

$$
z(x, y)=a(x, y) \exp [j \phi(x, y)]+\eta(x, y)
$$

where $\eta$ represents the noise term. The above equation can be represented as a stack of 1-D signals as:

$$
z_{y}(x)=a_{y}(x) \exp \left[j \phi_{y}(x)\right]+\eta_{y}(x) \text { for } x, y=1, \ldots, N
$$

where $y$ denotes the index of the row, $a_{y}(x)$ the amplitude of the $y^{\text {th }}$ row, $\eta_{y}(x)$ the additive complex white Gaussian noise (ACWGN) with zero-mean and variance $\sigma^{2}$, and $N \times N$ is the dimension of the fringe pattern. Since the interference phase to be estimated in holographic interferometry is, in general, a continuous function of the spatial coordinates, it can be approximated arbitrarily closely, in accordance to Weierstras approximation theorem (18), by a polynomial of a sufficiently high order. Thus Eq.(6) can be rewritten as:

$$
z(x)=a(x) \exp \left[j\left(\sum_{q=0}^{M} a_{q} x^{q}\right)\right]+\eta(x)
$$

where $q$ is an integer and $M$ is the degree of the polynomial. The subscript $y$ is dropped for the sake of simplicity. The problem of estimating the phase $\phi(x)$ from the noisy observations of $z(x)$ basically amounts to estimating the parameters like polynomial coefficients $\left\{a_{q}\right\}$ from $z(x)$. In order to estimate the polynomial coefficients from $z(x)$, a function $P T_{M}[z, \omega, \tau]$, popularly known as high-order ambiguity function (13) is used.

The $M^{t h}$ order ambiguity function of $z(x), P T_{M}[z, \omega, \tau]$, is defined as the Discrete Fourier transform of $P_{M}[z(x), \tau]$ :

$$
P T_{M}[z, \omega, \tau]=\sum_{x=(M-1) \tau}^{N} P_{M}[z(x), \tau] \exp (-j \omega x)
$$

where the function $P_{M}[z(x), \tau]$ is defined as:

$$
P_{M}[z(x), \tau]=\prod_{q=0}^{M-1}\left[z^{\dagger q}(x-q \tau)\right]\left(\begin{array}{c}
M-1 \\
q
\end{array}\right)
$$

where $z^{\dagger q}(x)= \begin{cases}z(x) & \text { if } q \text { is even } \\ z^{*}(x) & \text { if } q \text { is odd }\end{cases}$

$(\cdot)^{*}$ denotes complex-conjugation, $\tau$ is a positive real number and $\left(\begin{array}{l}x \\ y\end{array}\right)=\frac{x !}{(x-y) ! y !}$.

The properties of HAF imply that applying the operator $P_{M}$ on the signal $z(x)$ transforms the signal into a single tone whose frequency, say $\omega_{0}(\tau)$, is directly proportional to the highest-order polynomial coefficient $a_{M}$ :

$$
a_{M}=\frac{\omega_{0}(\tau)}{M ! \tau^{M-1}} \text { for all } \tau>0
$$




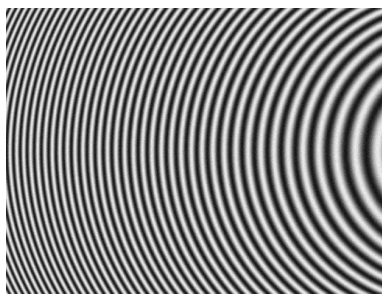

(a)

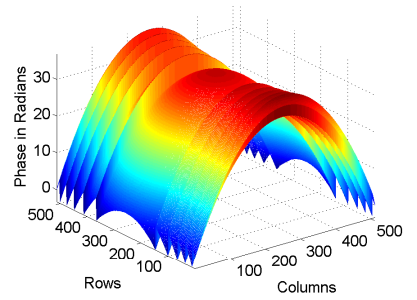

(d)

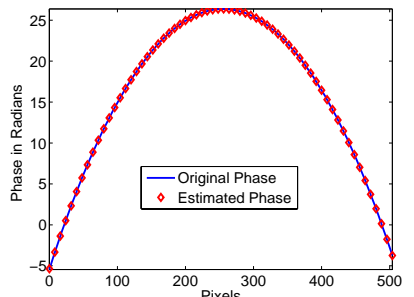

(b)

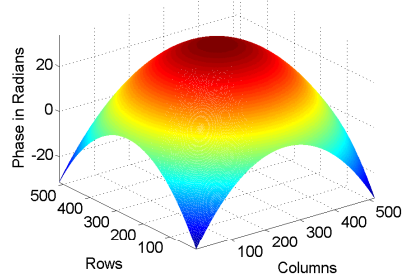

(e)

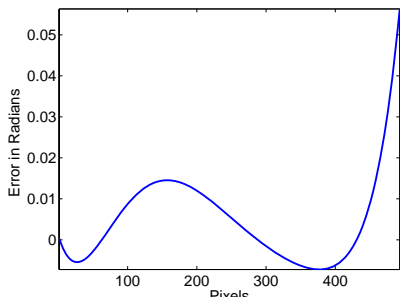

(c)

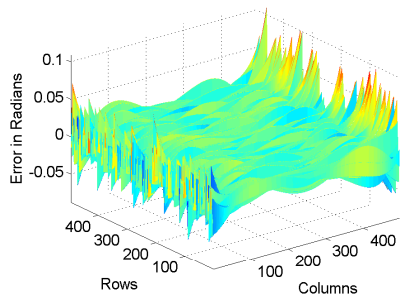

(f)

Figure 1.: (a) Simulated fringe pattern at $S N R$ of $30 d B$, (b) Phase estimated using the HAF method along the middle row after subtracting the carrier phase, (c) Error plot, (d) 3-D plot of the estimated phase along all rows (e) 3-D plot of the resulting phase map after phase stitching, (f) 3-D plot of the error distribution.

Based on this, the following procedure can be used to estimate the polynomial coefficients $\left\{a_{q}\right\}$ : First, $P_{M}$ of the signal $z(x)$ is computed; which produces a single tone signal. By calculating its frequency, say by identifying the peak in the FFT spectrum, the highest-order polynomial coefficient $a_{M}$ can be estimated using the relation in Eq.(10); let $\hat{a}_{M}$ denote the estimate of $a_{M}$. The effect of the highestorder phase term is then removed by "peeling-off" the signal as $z^{\prime}(x)=z(x)$. $\exp \left(-j \hat{a}_{M} x^{M}\right)$. The new signal $z^{\prime}(x)$ thus formed is a polynomial phase signal of the order $(M-1)$. Computing $P_{M-1}$ of $z^{\prime}(x)$ results in a single tone signal whose frequency is directly proportional to $a_{M-1}$. This process of estimating the highestorder coefficient in each iteration and peeling-off the signal to reduce its polynomial phase order is repeated until all the coefficients are estimated. Constructing a polynomial with these estimated coefficients results in the direct estimation of the unwrapped phase. Although the phase estimated along individual rows in this manner is unwrapped, to obtain a continuous 2-D phase distribution one additional operation of the type needed to weave all the rows to fall in line one after the other is required. We refer to this operation as phase stitching.

In the above process, the choice of $\tau$ affects the accuracy of estimated coefficients. It is shown in (13) that, mean square error of $a_{M}$ achieves a minimum at $\tau=\frac{N}{M}$ for $M=2,3$ and $\tau=\frac{N}{M+2}$ for $M \geq 4$.

\section{Simulation and Experimental Results}

Fig. 1a shows the image of a simulated fringe pattern. The discrete time analytic signal of the fringe pattern is computed using the Hilbert transform (17). Then the HAF-based approach is used for estimating directly the unwrapped phase distribution. In these simulations, accurate phase estimation is accomplished by using the fourth order polynomial phase signal model. Fig. 1b shows the profile of the estimated phase along the middle row, after eliminating the carrier phase. Fig. 1c shows the error plot. 3-D plot of the estimated phase over all the rows is shown in 


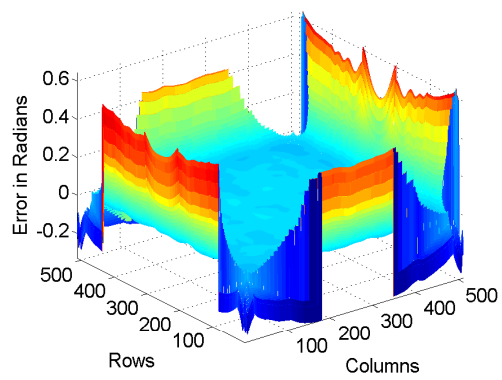

(a)

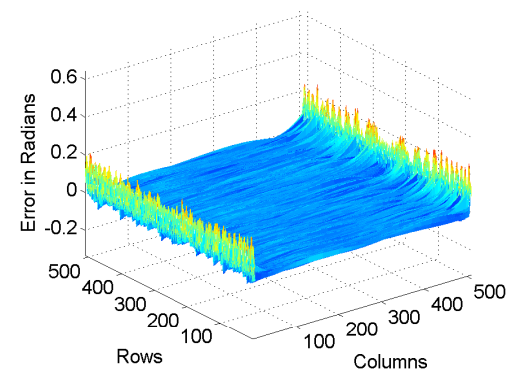

(b)

Figure 2.: Error plots when continuous phase distribution of Fig. 1a is estimated with (a) WFR method (b) HAF method

Table 1.: Performance comparison

\begin{tabular}{lcc}
\hline Method & RMSE & Time $(\mathrm{Sec})$ \\
\hline WFR & 0.0234 & 399 \\
HAF & 0.0121 & 15 \\
\hline
\end{tabular}

Fig. 1d. As is clear from the previous figure, phase stitching is needed to obtain a continuous 2-D phase distribution. However, it is as easy as 1-D unwrapping of an ideal wrapped phase map. Fig. 1e shows the 3D plot of the interference phase estimated with the HAF method after phase stitching. Error in estimation is shown in Fig. 1f.

Note that RPT method (12) also provides directly the unwrapped phase distribution from the normalized fringe pattern (Eq.(3)); if Eq.(5) is the input, both RPT of Servin (19) and windowed Fourier ridge (WFR) of Kemao (3, 20) will produce accurate phase estimation. In (21) Kemao et al. have provided an elegant comparative analysis of five effective wrapped phase filtering techniques, which included RPT and WFR. Here we compare the results of the HAF-based method with WFR. Fig. 2 shows 3-D mesh plots of error distributions when continuous phase distribution of the pattern shown in Fig. 1a is estimated with both the methods. As is evident from Fig. 2, WFR method produces results with relatively large errors near the edges of the image. Although the HAF method shows to an extent a similar tendency, it produces relatively small errors. For example, if all pixels of the fringe pattern are considered, the root mean square errors (RMSE) produced by WFR and HAF methods are given by 0.0794 and 0.0208 , respectively. For this reason, 10 rows from both top and bottom and 10 columns from both left and right boundaries of the estimated phase are not considered (size of the window used in WFR algorithm is 10x10) either while calculating the RMSE values listed in Table 1 or displaying the error plot in Fig. 1. The RMSE and computational cost for HAF and WFR methods are listed in Table 1. These tests are run on a Windows PC with Intel Core 2 Duo processor of $2.66 \mathrm{GHz}, 3.24 \mathrm{~GB}$ of RAM and with MATLAB 7.0.4. version. It is evident that the HAF method provides accurate results with relatively less computational time.

Fig. 3a shows the comparison of error plots when the phase along a row is estimated with the HAF method by approximating the phase of the fringe pattern with polynomials of different orders (at $\mathrm{SNR}=30 \mathrm{~dB}$ ). We have performed error analysis at different SNRs with fourth order polynomial approximation, results of which are shown in Fig. 3b.

The experimental results shown in Fig. 4 substantiate the applicability of this 


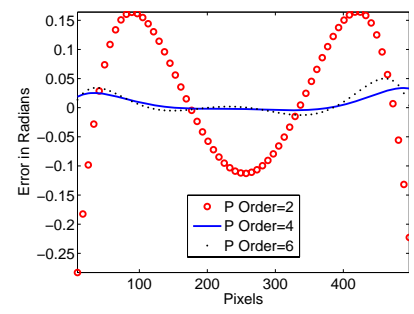

(a)

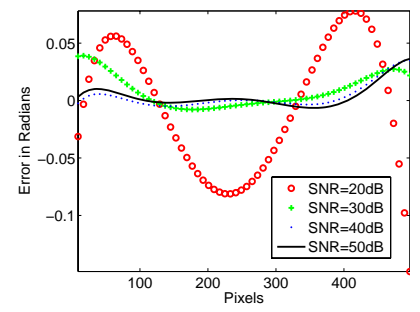

(b)

Figure 3.: Error plots when (a) Approximated with different orders of polynomials at SNR of $30 \mathrm{~dB}(\mathrm{~b})$ Approximated with a 4th order polynomial at different levels of SNR

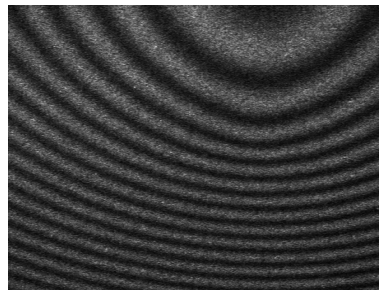

(a)

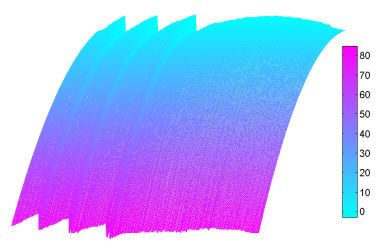

(b)

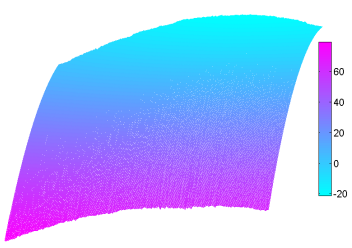

(c)

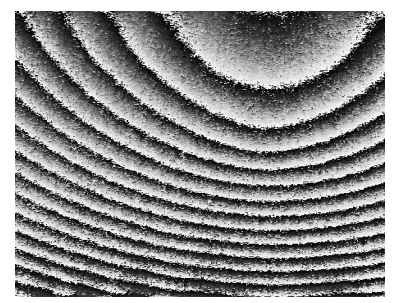

(d)

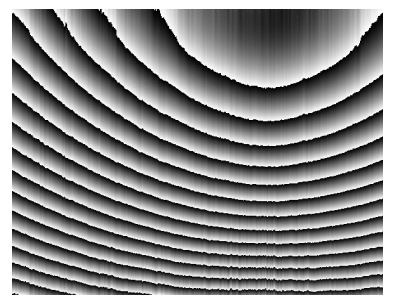

(e)

Figure 4.: (a) Experimentally recorded holographic fringe pattern, (b) 3-D plot of the estimated phase along all rows using HAF method, (c) 3-D plot of the resulting phase map after phase stitching, (d) Phase calculated directly from the analytic signal using arctan function, (e) Generated wrapped phase map from the continuous phase distribution in Fig.4c.

method for phase estimation in holographic interferometry. For the sake of comparison, Fig. 4d and Fig. 4e show, respectively, the phase map calculated from the analytic signal using arctan function and the wrapped phase generated from the estimated continuous phase (Fig. 4c) obtained using the proposed method.

\section{Conclusions}

This Letter introduced a new approach for the analysis of fringe patterns recorded in holographic interferometry. The presented HAF-based analysis approach is capable of providing an accurate estimation of the phase map from a single fringe pattern. The simulation and experimental results illustrate the ability of the HAFbased analysis approach in directly estimating the unwrapped phase distribution. In addition, it is capable of analyzing fringe patterns having large frequency variations. 


\section{Acknowledgement}

This work is funded by Swiss National Science Foundation under Grant 200020113196 .

\section{References}

(1) Takeda, M.; Ina, H.; Kobayashi, S. J. Opt. Soc. Am. 1982, 72 (1), 156-160.

(2) Kemao, Q. Appl. Opt. 2004, 43 (13), 2695-2702.

(3) Kemao, Q. Opt. Las. Engg. 2007, 45 (2), 304-317.

(4) Zhong, J.; Weng, J. Opt. Engg. 2004, 43 (4), 895-899.

(5) Afifi, M.; Fassi-Fihri, A.; Marjane, M.; Nassim, K.; Sidki, M.; Rachafi, S. Opt. Commun. 2002, 211 $(1-6), 47-51$

(6) Dursun, A.; Ozder, S.; Ecevit, F.N. Meas. Sci. Tech. 2004, 15 (9), 1768-1772.

(7) Zhong, J.; Weng, J. Appl. Opt. 2004, 43 (26), 4993-4998.

(8) Gdeisat, M.A.; Burton, D.R.; Lalor, M.J. Appl. Opt. 2006, 45 (34), 8722-8732.

(9) Federico, A.; Kaufmann, G.H. Opt. Engg. 2002, 41 (12), 3209-3216.

(10) Federico, A.; Kaufmann, G.H. Applied Optics 2003, 42 (35), 7066-7071.

(11) Sciammarella, C.A.; Kim, T. Opt. Engg. 2003, 42 (11), 3182-3193.

(12) Servin, M.; Marroquin, J.L.; Cuevas, F.J. Appl. Opt. 1997, 36 (19), 4540-4548.

(13) Peleg, S.; Friedlander, B. IEEE Trans. on Sig. Process. 1995, 43 (8), 1901-1914.

(14) Ochoa, N.A.; Silva-Moreno, A.A. Opt. Commun. 2007, 270 (2), 161-168.

(15) Guerrero, J.A.; Marroquin, J.L.; Rivera, M.; Quiroga, J. A. Opt. Lett. 2005, 30 (22), $3018-3020$.

(16) Quiroga, J.A.; Servin, M. Opt. Commun. 2003, 224 (4-6), 221-227.

(17) Lawrence Marple Jr., S. IEEE Trans. on Sig. Process. 1999, 47 (9), 2600-2603.

(18) Rudin, W. Principles of mathematical analysis. Third Edition, McGraw-Hill Sci. Engg., NY, 1976.

(19) Servin, M.; Cuevas, F.J.; Malacara, D.; Marroquin, J.L.; Rodriguez-Vera,R. Appl. Opt. 1999, 38 (10), 1934-1941.

(20) Qian, K.; Seah, H.S.; Asundi, A. Opt. Engg. 2003, 42 (10), 2792-2793.

(21) Kemao, Q.; Nam, L.T.H.; Feng, L.; Soon,S. H. App. Opt. 2007, 46 (30), 7412-7418. 